\section{Praveen Chaudhari}

I think there is a world market for

maybe five computers.

—Thomas Watson, Chair of IBM, 1943

$640 \mathrm{~K}$ ought to be enough for

anybody.

—Bill Gates, 1981

It is difficult, if not impossible, to visualize what the world will look like in the decades ahead. If current developments are any guide, changes brought about by information technology are likely to be deep, pervasive, and rapid. They will span the world. Every activity associated with humans can be expected to be increasingly affected by the almost instant availability of a worldwide pool of information coupled with our ability to extract and, if need be, process this information for a very wide variety of uses. Those parts of the world that do not adopt and adapt to these rapidly evolving, some say revolutionary, changes will surely be marginalized.

Technologies associated with the communication, processing, and storing of information in primarily binary form are collectively called information technology (IT). Also included in IT are human/ machine interface technologies. This article is concerned only with the physical aspects of IT, namely, hardware used to process, store, transmit, and present information. In such a rapidly moving field, "incremental" changes associated with known technologies will cause radical changes in the next 15 years. Such developments in areas of communication technologies, human/machine interaction, storage technologies, and memory/microprocessor/ ASIC/systems-on-a-chip, are covered in this article, describing the current status, nearterm projections, and future scenarios. But this article also veers off of the various roadmaps, projecting developments far into this new century and stretching our imaginations to intriguing possibilities such as using DNA and DNA-like molecules in information technology.

It is useful to consider the monetary aspects of IT hardware, so as to view its financial import in some perspective. In the near future, a personal communication system (PCS), which is the evolving cell phone, is likely to cost on the order of $\$ 100$, and a home computer with a large display that can also serve as an entertainment center, about $\$ 900$. Assuming that these are the only two devices an average family owns and, assuming further, that only a fraction of the world's population owns them, say a billion people, the annual turnover is about a trillion dollars. If we add to this the IT hardware needs of institutions and the economics associated with software, systems, and applications, it is easy to see that the IT industry is likely to be a muti-trillion-dollar business and a dominant force shaping trade between nations.

Unlike natural resources that are determined by location, such as oil, IT is in principle not bound to particular areas of the globe. It requires know-how and determination, which are widely available. However, the infrastructure and financial resources needed-which can be very large- are often lacking. Despite these limitations, the present growth of technology, limits to miniaturization, and still-evolving standards offer unique opportunities for new players to enter the field.

IT hardware development has been characterized by a sustained and historically unprecedented rate of growth. For example, the bandwidth in communication systems, microprocessor speeds, and memory and storage densities have all doubled every 18-24 months for the last three to four decades. Along with performance enhancements, the cost per elementary bit transaction, such as speed or density, has also decreased exponentially. Many factors are responsible for this. From the narrow perspective of technologists, this has been possible because of the tight coupling between research, development, and manufacturing. From an even-narrower perspective of research, which is the subject of this article, it is the sustained developments in materials research that have provided the underpinning of a remarkable three decades of progress.

\section{Communication Technologies}

It is widely believed that in the future a human being will have the ability to be electronically connected to any other human being and to information sources scattered throughout the world; the connection will include both audio and video

Materials Challenges For The Next Century presents a series of articles speculating on the role of materials in society in the coming century and beyond. capabilities. This will require large-bandwidth communication links from point to point. Given the wide diversity of economic development of nations and of geography, both space- and terrestrial-based systems are likely to be used. When speed of communication is the primary consideration, as when transmitting or accessing large databases over long distances, a fiber-optic system is the preferred mode. This advantage over wireless communication is associated primarily with the increased absorption of radiation by water and air as the wavelength of the wireless radiation is reduced.

\section{Wireless}

The demands for new or improved materials in communication technology are numerous. Let us look at the wireless world first. The PCS cannot be a highpowered device because of safety considerations, as it is used close to the human body. For example, the current and near-term devices operate at a frequency of $1-2 \mathrm{GHz}$, and the power output is a watt or less. If satellites are the principal means of wireless communication, it is not readily feasible for a geosynchronous satellite at an altitude of $36,000 \mathrm{~km}$ to receive a $1-\mathrm{W}$ signal, amplify it, and transmit it to a PCS at the other end of the world. Much larger antennas than currently used and increased power generation are required. A typical geosynchronous satellite needs kilowatts of power and, for it to be useful for a PCS, the power availability has to increase between one and two orders of magnitude. Thus solar cells of still-higher efficiency but manageable cost are essential.

Due to the available power constraints, medium-orbit $(10,000 \mathrm{~km})$ and low-orbit $(1,500 \mathrm{~km})$ satellite systems have been proposed. The antenna size and power needs are reduced, as they change inversely with the square of the orbital distance from the source. However, the number of satellites required, and the associated cost, also changes. Three or four satellites placed at the geosynchronous orbit provide adequate worldwide coverage, whereas medium orbits require about 12 units and low orbits about 50 units. In spite of the increased cost, several corporations are actively pursuing these alternative approaches to the geosynchronous placement of satellites because of the anticipated demand for high-speed and large bandwidth communication channels for worldwide applications. 
High-speed but local wireless links for PCSs are also expected to be in wide demand. Both cost and performance become primary considerations. This is in contrast to military applications, where performance can often be the primary concern. Both the PCS and base stations have a need for high-performance digital, analog, and rf circuits. Progress in this area is, in part, gated by the availability of low-cost but high-frequency substrate materials. Laminates of various kinds and low-temperature multilayered ceramic substrates are current contenders. GaAs, from which MESFETs and HBTs have been fabricated for communication circuits, has been the material of choice. However, SiGe HBTs are now being manufactured using BiCMOS technology. Using $0.5-\mu \mathrm{m}$ technology, devices operating in the range of $50-60 \mathrm{GHz}$ have been produced. In principle, the SiGe technology has two advantages over GaAs: integration of analog and digital circuits on the same silicon chip (for system-on-achip applications) and the very large base of manufacturing know-how developed for the mainstream Si technology used in computers. In base stations, HTSs are being considered for passive high- $Q$ filter applications. If this happens, it is likely that this will be the first large-scale application of these materials. Although commercial HTS SQUIDs are available, their market value is relatively small. Superconducting wires, although they are very promising, are still in the development stage. The HTSs are used in filter applications for analog circuits, where they offer a high degree of frequency selectivity in a relatively compact size.

\section{Cables}

The attenuation of light of modern silicabased-glass fiber-optic cables is between 0.1 and $10 \mathrm{~dB} / \mathrm{km}$, depending upon the wavelength of light, usually around 1 or $1.5 \mu \mathrm{m}$. Plastic fibers, which are cheaper and some- what easier to handle, are also used, though primarily in local-area networks because of their high attenuation rates, approximately $200 \mathrm{~dB} / \mathrm{km}$. Hollow (glass) fibers can transmit radiation from the visible to the far-infrared. These fibers are primarily used in medical applications as sensors. Even though the attenuation of light by high-quality glass fibers is small, it is not zero. Hence the signal has to be periodically amplified. Erbium-doped fibers are used to amplify optical signals, without converting them first from optical to electronic and then, after amplification, back again to optical. In general, optical amplifiers and lasers, used as amplifier pumps, are an active area of materials research, both for silica glass and plastic fibers.

Future high-speed
detectors operating in the
range of $40-100 \mathrm{GHz} / \mathrm{s}$ bit
rate are conceivable.

Using wavelength division multiplexing, optical-transmission systems operating at hundreds of gigabits per second are available commercially, and laboratory demonstrations of signal-transmission rates of several terabits have been made. For these high data rates, the electronic circuitry used for detection and rerouting a signal is the limiting step for high-speed networks. Optical switching devices, using nonlinear optical materials, have been proposed and successfully demonstrated. However, these are still in the development stage, and new, nonlinear materials and their processing are desirable for future mesh-network topology. An alternate approach to a purely nonlinear optical material is to use a semiconductor optical amplifier biased such that an incoming optical signal leads to stimulated emission. The change in refractive index caused by inversion-filling of the valence band is very large and can be used for demultiplexing. The area of optoelectronic materials for use in severalterahertz networks is and will remain, in the foreseeable future, a very active area of research and development.

Assuming that a local optical signal has been obtained from a multiplexed fiber cable, it has to be detected by an electronic circuit before it can be used. The speed of such front-end detectors is still in the $\mathrm{GHz} / \mathrm{s}$ bit rate. Based on what we know, future high-speed detectors operating at a bit rate in the range of $40-100 \mathrm{GHz} / \mathrm{s}$ are conceivable. They will require optimally designed optoelectronic integrated circuits (OEICs). Photo detectors operating in the range of $100 \mathrm{GHz}$ have been made, as have amplifiers. The photo detectors are made of InGaAs, which is lattice-matched to an InP substrate. InGaAs is the lightabsorption media, and the detector geometries have a $p-i-n$ arrangement, with light usually incident on the $p$-side. Metal-semiconductor-metal diodes have also been used, where the semiconductor can be GaAs. Typical amplifiers use either the HBT or the HFET. The transistor materials are typically $n$-AlInAs/GaInAs, deposited on an InP substrate. The common InP substrate allows for monolithic integration between the detector and amplifier.

Materials for high-speed communication appears to be a fertile field for research because of the potential for innovation. There are several competing alternatives to providing a worldwide communication system, and discoveries in materials may decide which alternative becomes the dominant mode of communication. The financial stakes are enormous.

\section{Human/Machine Interaction: Display Technologies}

The relatively cheap and bulky, yet venerable, CRT will be replaced in the home by a high-definition digital televi-

\begin{tabular}{|ll|}
\hline & ACRONYM KEY \\
AMLCD: active-matrix liquid-crystal display & LCD: liquid-crystal display \\
BiCMOS: bipolar and complimentary metal oxide semiconductor & MEMS: microelectromechanical systems \\
CRT: cathode-ray tube & MO: magneto-optic \\
CVD: chemical vapor deposition & MESFET: metal-semiconductor field-effect transistor \\
dlc: diamondlike carbon & MOSFET: metal-oxide-semiconductor field-effect transistor \\
DRAM: dynamic random-access memory & OEIC: optoelectronic integrated circuit \\
FED: field-emission display & OLED: organic light-emitting diode \\
GMR: giant magnetoresistive & PCS: personal communication system \\
HBT: heterojunction bipolar transistor & rf: radio frequency \\
HFET: heterostructure field-effect transistor & SoC: system-on-a-chip \\
HTS: high-temperature superconductor & SQUID: semiconducting quantum interference device \\
IT: information technology & UV: ultraviolet \\
\hline
\end{tabular}


sion screen that will be flat and probably will cover a wall or a good fraction of it. This much appears to be inevitable. However, even though ideas on how to realize this are well established, no single technology is viewed as a sure winner. Most, if not all, of the issues are materialsand process-related. Therein lie the opportunities for materials researchers. In the following list of display-technology ideas and related materials issues is the accompanying need to develop a manufacturing process that is cost-effective.

\section{Plasma Display}

In plasma displays, which currently dominate the large-display end, a plasma is generated in a gas such as helium or xenon, much as one does in a fluorescent tube for room lighting. The tubes used in the plasma display are hundreds of microns in size, and depending upon the size of the display, 10 million of them are present in a single display. The plasma generates UV radiation, which then excites a red, blue, or green phosphor. The small tubes are arranged in arrays, and a matrix address system is used to address a particular pixel, comprising three small tubes containing the three colors. As a result, the luminance and resolution of plasma displays are very uniform across the entire area of the display. This is in contrast to a CRT, which loses both resolution and luminance at the edges and corners. The projection displays (discussed later) also suffer from this limitation. A drawback of the matrix address system is that the number of drive circuits scales with the number of elements.

Another drawback is the need for lithographic systems that can expose large areas with the requisite resolution and alignment capabilities. Clearly, if the lithography step can be eliminated or minimized, there would be large cost savings. A cursory look at this problem does not offer much hope of eliminating or minimizing the lithography. However, materials expertise might be useful. The size of the pixel is relatively large, and it may therefore be possible to deposit materials directly through a mask below which the substrate is scanned. It may also be possible to use well-controlled phase separation and selective etching to produce the tiny plasma tubes. I mention these two possibilities only as an illustration of the opportunities for materials researchers to use their imaginations to overcome the many cost hurdles that are present in this and the display technologies described next. More conventional materials issues in this technology are the life of the phosphors, more efficient phosphors, and the design of a plasma cell such that the sputtered atoms do not deposit on the phosphor or short the electrodes.

\section{Active-Matrix Liquid- Crystal Display \\ AMLCD is} another technology migrating toward large sizes. This technology is pervasive in computer laptops and is making inroads into the desktop market. A liquid-crystal molecule is rod shaped, about 2 $\mathrm{nm}$ in length, and $0.5 \mathrm{~nm}$ in diameter. It is optically birefringent and aligns itself either parallel or perpendicular to an electrical field depending upon the sign of its dielectric constant. The anisotropic optical and electrical properties make it useful for display applications. Liquid crystals can be aligned (i.e., made parallel to a given direction) by rubbing the substrate they are on. The liquid crystal is usually deposited on a polymer such as polyimide. This is rubbed with a velvet cloth. One of the "dreams" of this technology has been to develop a noncontact alignment process. Rubbing introduces debris in an otherwise clean manufacturing process and can damage the transistors below the polyimide layer by the discharge of electrostatic fields. The process also leaves streaks on the surface, which can produce a poor "front of screen" quality.

\section{Liquid-Crystal Displays}

LCDs are often characterized by alignment or, alternatively, by the arrangement of the electric fields used to switch the direction of a liquid-crystal cell. The most common mode is the twisted nematic used in laptop computers. In this, the two surfaces of the cell enclosing the liquid crystal are rotated by $90^{\circ}$, and hence the liquid-crystal molecules are twisted by $90^{\circ}$ as they conform to the boundary conditions imposed by the two interfaces. If this cell is inserted between two polarizers that have been crossed, it transmits light. Alternatively, if a voltage is applied across the cell, the liquid-crystal molecules rotate and align parallel to the electric field, and no light is transmitted by the crossed polarizers. Hence an electric field switches the light on and off (sometimes called a light valve by the display community). As in plasma displays, pixels comprised of three colors are arranged in arrays and addressed individually. The electrical field for switching is provided by charging a capacitor plate made of indium tin oxide (an optically transparent conductor) and placed below the polyimide layer in each cell. The curent to the capacitor is controlled by a transistor, and each cell has its own transistor. Hence each pixel in a good resolution display is about $200 \mu \mathrm{m}$ by $200 \mu \mathrm{m}$ or less and contains three transistors.

Materials and processing issues control the cost of these displays. For example, can one align the liquid crystals locally such that each pixel has effectively two or preferably four different alignment directions? If this could be done economically, then the "angle of viewing" of the display would compare favorably with the CRT or the plasma display. The angle-of-viewing problem is very obvious in laptop displays, requiring the display angle to be adjusted relative to the position of an observer. One way around this is used in desktop displays. The twisted nematic arrangement of the liquid crystal is replaced by a parallel alignment, and inplane electric-field switching is used to turn the light on or off. This gives a much better viewing angle than the twisted nematic, but requires more power, which limits its use in battery-operated laptops.

The materials issues in LCDs range from suitable glass or plastic substrates, transparent electrodes, materials for transistors, alignment techniques, liquid crystals, and color filter materials to topological defects such as disclinations in liquid crystals and their optical properties. Apart from alignment and multidomain generation, transistor materials is a very active area of research. Currently, these are made of hydrogen-doped $(\sim 10 \%)$ amorphous silicon deposited from, say, $\mathrm{SiH}_{4}$ by various CVD techniques. Transistors made from amorphous Si are limited by the mobilities of this material $\left(\sim 1 \mathrm{~cm}^{2} / \mathrm{V} \mathrm{s}\right)$. Polycrystalline silicon outside the grain-boundary area has much 
higher mobilities. Like the grain boundaries in a variety of materials, such as the HTSs, the electrical properties of silicon grain boundaries are undesirable for transistor applications. Several schemes are being explored for producing single-crystal or controlled grain-boundary-location polycrystalline silicon to replace amorphous silicon. The most discussed approach has been to use a pulsed excimer laser beam to crystallize amorphous silicon on a glass substrate. Also, a number of organic materials are being explored as alternatives to silicon.

\section{Electroluminance Display Devices}

In electroluminance display devices, electron-hole recombination occurs by radiative decay in the visible. The device, in its simplest form, is composed of three layers: two electrodes (one of which is optically transparent) and an electroluminescent material. The historical phosphor for this application is $\mathrm{ZnS}: \mathrm{Cu}$, which emits the familiar green light. Many phosphors have been developed to produce yellow, green, red, and blue emissions. Practical devices usually have two additional insulating layers adjoining the luminescent material. These layers enhance the efficiency of injecting ballistic electrons into the luminescent material. The thickness of the three layers is controlled by the electrical breakdown strengths and their dielectric constants. These devices have high-contrast displays, wide viewing angles, and long lifetimes. A large number of luminescent materials have been developed to provide the red, green, and blue emissions necessary for a color display.

One of the "dreams" of
[AMLCD] technology has
been to develop a noncontact-
alignment process.

Starting in the 1960s, but particularly within the last decade, organic and conjugated polymeric materials have been extensively investigated for electroluminescent devices. This was primarily due to the discovery that the use of multilayer organic films reduced the operating voltages and enhanced the quantum efficiency of light emission. Since then, the lifetime of operation of these devices has continued to improve, along with the quality and control of the wavelength of emission. These devices rely on radiative decay from an exciton produced by electrons and holes injected into the luminescent film or interface between hole- and electroncarrying films. The principal advantage of the organic and polymeric films is the ease of deposition of films over large areas. Another advantage cited for the use of these materials is that the organic dye materials can frequently be used in polymer dispersed systems, thus providing for the possibility of using printing technology or ink-jet technology to fabricate displays. A number of hole- and electrontransport organic materials have good thermal stability. For example, aromatic polyamines and high-molar-mass aromatic amines show improved thermal stability in multilayer structures as holetransport layers. Tris (aluminum) $\left(\mathrm{Alq}_{3}\right)$ is a very good electron-transport material.

OLEDs have progressed to the point where they appear to be a viable alternative to the inorganic-materials-based devices and, more important, to LCDs. The organic materials are easier to deposit than the inorganic materials and have a higher luminosity and a larger viewing angle than the current liquidcrystal devices. However, the luminosity is still below that of a conventional CRT. Some of the challenges in these materials lie in increasing their efficiencies, particularly in the red and blue emissive materials. Another issue is obtaining all three colors with electrical parameters that match each other, otherwise the drive circuitry has to be adjusted to each color, thus increasing cost.

\section{Field Emission}

Another display technology that is being actively researched is based on field emission from a microscopic array of etched points, such as in silicon, or alternatively of dots made of low-work-function materials, such as diamondlike carbon (dlc) films. The emitted electrons travel through a vacuum to strike a phosphor at about $100 \mathrm{eV}$. FEDs are essentially a low-voltage, flat-panel version of the standard CRT. An active area of research is the development of suitable low-voltage phosphors and an inexpensive technology to produce a large and controllable array of field emitters. The relatively high voltage required to drive these devices has been suggested to be a constraint to the use of this technology because the reactive power required to drive these devices goes as the voltage squared.

\section{Projection and "Electronic Ink" Displays}

The projection displays use either liquidcrystal light valves or an array of nanoscale mirrors produced in a silicon wafer. The light valve and the mirrors are driven by silicon devices integrated into the display. The electronic ink or paper contains microencapsulated particles in the range of $100-1000 \mathrm{~nm}$. The particles are highly scattering or absorbing and migrate in electric fields. Electrophoretic materials have also been proposed for electronic paper applications. Microparticle-based displays have high contrast and reflectivity and a nearLambertian viewing characteristic. This field is still evolving, and the potential for new discoveries is very good.

The display area, excluding our extant television sets at home or the CRT monitors on our desktops, has always been underestimated in its sophistication of research and potential for financial impact. It is a business that is currently worth many billions of dollars and is expected to grow many fold as new display technologies move from laptops to desktops to large screens and, as the cost of manufacturing comes down, toward the home. Materials and manufacturing are the key drivers.

\section{Disk-Storage Technologies Magnetic Storage}

While, historically, information has been stored on tapes and disks, disk-storage technologies represent the bulk of the anticipated growth in the future. The diskstorage industry is valued at $\sim \$ 50$ billion and growing at a rate of $\sim 20 \%$, even though magnetic disks have been used for over four decades. Disk-storage technologies include the familiar read-only compact disks, used widely in the home-entertainment business, and read-and-write magnetic, magneto-optic, and phasechange disks. Of these, I shall not say anything about the compact disk beyond the fact that it uses changes in reflectivity to store data in patterned, metallized films deposited on plastic substrates. I shall, however, discuss holographic and scanning-probe-based storage ideas.

Magnetic disks continue to be the mainstay of storage technology. Until about nine years ago, essentially the same technology introduced decades ago was still being used: The direction of magnetization on a film was changed locally by bringing a head close to the surface of the film and applying a pulse of a magnetic field, using an electrical current pulse through a suitable coil. The head was designed to stay above the spinning disk, using air as a bearing. A lubricant film was placed on top of the magnetic media on the substrate to reduce friction. The information, written into the magnetic media as a change in the direction of magnetization, was read by a signal induced in a coil, also placed on the head. 
The first significant change in materials technology was in the head design. Instead of an inductive readout, a magnetoresistive sensor was introduced. This is being followed by a GMR sensor and a spin valve, and research is under way to see if a colossal magnetoresistance sensor can be developed. These changes in sensors are essential to maintain the rate of progress in storage densities. The magnetic films deposited on the disk are also changing. Thin smooth films are replacing the particulates of the past. The change in magnetic flux above the disk decreases more rapidly as the bit gets smaller. To compensate for this, the spacing of the head from the disk surface has to be decreased. Even the design and materials used in the write head are likely to change in the near future, as the frequency of operation of the write head is pushed to higher values.

The accelerated growth rate $(\sim 60 \%$ per year for the last decade) in the density of storage has been possible because of the change from inductive to magnetoresistive heads. As the density of storage increases, the stray field, used to sense a written bit, decreases for a given height above the surface of the media. To compensate for this decreasing field, the inductive heads were made more sensitive by increasing the number of turns in the inductive head. This increased the resistance of the head and the associated thermal noise, hence limiting the storage density to about a gigabit per square inch. This limitation was overcome by using a magnetoresistive head. This and the GMR heads rely on a change in the resistance of the material as the magnetic field is switched from one direction to another. In the early magnetoresistive heads made of permalloy, $\mathrm{Ni}_{80} \mathrm{Fe}_{20}$, the change in resistance was $\sim 2 \%$, and the corresponding voltage signal was several times larger than in inductive heads. Also, unlike the inductive heads, whose signal is proportional to a change in the rate of flux, the magnetoresistive-head signal is proportional to flux. In the late 1980s, it was discovered that multilayer materials, such as those of $\mathrm{Fe}$ and $\mathrm{Cr}$, produced changes in magnetoresistance of the order of $40-50 \%$ (and hence the term "giant" magnetoresistance). Typically, the Fe layer is about $3 \mathrm{~nm}$ thick and the $\mathrm{Cr}$ layer about $1 \mathrm{~nm}$ thick. The Fe couples antiparallel to $\mathrm{Cr}$. In an applied field, the spins of the soft iron layers are switched parallel to the $\mathrm{Cr}$. This switching changes the interface scattering of the charge carriers in Fe to produce large changes in resistance. Practical devices, called spin valves or GMR, rely on this physical effect, but use materials in which the switching can be accomplished at

lower fields. The increased signal has enabled the density of storage to increase and push the limits to media rather than head noise. In recent developments, manganesebased perovskites have been found to have a magnetoresistance change much larger than that of the giant magnetoresistance. These materials were dubbed as having colossal magnetoresistance. Unfortunately, the large values of magnetoresistance are currently observed only below room temperature.

\section{In human terms, a single disk with a petabit of storage provides approximately a movie a day for over 60 years.}

If the last decade has been characterized by developments in head materials, the current and near future will be characterized by the development of storage media, including the magnetic and wearresistance layers. Cobalt alloy films containing $\mathrm{Cr}$ are the magnetic films of choice for media. The cobalt alloys have high coercivity associated with a large moment and magnetic anisotropy. The films are deposited such that the grain size of the films is small, in tens of nanometers, and the $\mathrm{Cr}$ segregates to the grain boundaries to minimize exchange coupling between the grains. An undercoating, such as thin Ti film, is used to align the $c$-axis of the Co grains in the plain of the film. These materials, called in-plane recording media because a bit of information is recorded by the reversal of magnetization in the plane of the media, are to be contrasted with future trends toward perpendicular recording, where the magnetization is perpendicular to the plane of the film. In in-plane switching media, recording noise is associated with the variations in the direction of the $c$-axis of different grains in the plane of the film that comprise a bit region. Clearly, better alignment is highly desirable, and for this reason, the perpendicular media has an advantage. However, this will require a redesign of the write and read heads. Another trend in media design is to use patterned substrates on which features a few tens of nanometers in spatial dimension have been fabricated, using relatively inexpensive lithographic means, such as embossing or laser interference combined with electron or x-ray lithography.

The magnetic films are usually covered by a thin film, of the order of $5 \mathrm{~nm}$ thick, of a hard coating such as nitrogen-doped amorphous carbon films, called diamondlike carbon (dlc). The term "diamondlike" arises from the presence of a large fraction of $s p^{3}$ bonds over the $s p^{2}$ bonds seen in graphite. The dlc film is then covered by a lubricating film about a monolayer thick. In order to maintain the rate of historical progress of the last decade, the distance from the head surface to the magneticmedia surface has to be reduced. This spacing, including the slider-air-bearing distance, is in the range of $10-20 \mathrm{~nm}$. The drive is to reduce this spacing further, even though there is not much left to play with. This imposes one limit; another more fundamental limit holds the maximum density of storage to be between 40 and 100 gigabits/in. ${ }^{2}$ This is associated with the paramagnetic limit reached as the size of the grain is reduced; thermal fluctuations at room temperature can overcome the magnetic anisotropy energy per unit volume.

\section{Magneto-Optic Storage}

Optical storage does not have the headto-media spacing constraint. In this technology, a laser beam is used to write and read a bit of information. Like the readonly optical compact disks, and unlike the high-density storage devices previously described, the read-write optical disks have an advantage in that they can be removed or inserted by the user. The bit size scales with the wavelength of light, although some schemes enable one to exceed this limit. In phase-change media, an amorphous film, typically a Tebased chalcogenide material, is crystallized by a suitably intense laser beam. A higher-intensity laser beam reverses the crystallization by melting the material, which then cools back to the amorphous phase. Information is read by measuring the reflectivity of the film; the optical reflectivity of the crystalline phase is different from that of the amorphous phase. The read-write media used increasingly in the entertainment industry favors this technology over the magneto-optic (MO) storage technology described next because of the lower cost-despite the fact that the number of cycles for writing and erasing are limited.

The first commercial read-write opticalstorage technology was developed using magnetic materials. A bit of information is stored in a $\mathrm{MO}$ film by using a laser beam to heat an amorphous rare-earthtransition-metal alloy film in an applied magnetic field. The size of the data bit, limited by diffraction, is determined by the wavelength of light and the numerical aperture of the lens in conventional MO disks. Hence compact and inexpensive short-wavelength lasers in the blue are highly desirable. The stored information 
is read using the same laser, albeit at a lower intensity, by monitoring the change in Kerr rotation of the polarized light incident on the MO film. The films contain rare earths such $\mathrm{Tb}, \mathrm{Gd}$, and $\mathrm{Dy}$, and the transition metals are $\mathrm{Fe}$ and Co. The alloys are antiferromagnetic, and the compensation point as well as the Curie point can be adjusted to obtain optimal device behavior. The sputtered films have a large perpendicular magnetic anisotropy even though the amorphous structure suggests otherwise. This anisotropy is essential for storage as the change in the direction of magnetization with the laser and applied magnetic field is perpendicular to the plane of the film.

The diffraction limit set by normal optics can be bypassed using near-field optics. Commercial development of $\mathrm{MO}$ technology with storage densities between 10 and 40 gigabits/in. ${ }^{2}$ are being explored. In this technology, a flying head, similar to the inductive recording heads, is used to house two optical lenses and a coil to provide a magnetic field. One of the lenses is used as a near-field lens that then defines a beam-spot diameter smaller than the limit set by conventional diffraction theory. Media similar to the current MO media have been shown to record bit sizes in the range of $10 \mathrm{~nm}$. If near-field $\mathrm{MO}$ recording, optical or otherwise, comes into commercial existence, it could pose a threat to the historically dominant inductive recording.

\section{Higher-Density Storage Technologies}

Regardless of the race between the two magnetic technologies discussed here, it is anticipated that even-higher densitystorage technologies well into the terabit/ in. ${ }^{2}$ range and perhaps approaching the petabit/in. ${ }^{2}$ range will be required and, I believe, achieved in practical systems. Two approaches have been taken toward this goal. In one, holographic storage, and in the other, arrays of scanning probes, are being developed to record and read bits of

\section{Extracted from the International Technology Roadmap} for Semiconductors:

1. Technology breakthroughs in materials and processes:

1.1 The use of physically thicker (than silicon dioxide) gate materials to minimize direct tunnel current through the MOSFET gate while maintaining highcapacitance (higher k).

1.2 The use of metal gate electrodes to compensate for slower processing speeds caused by depletion in poly-silicon electrodes and boron penetration of the silicon substrate (from the poly-silicon).

1.3 Methods for forming ultra-thin and low-sheet-resistance pn junctions for higher performance transistors.

1.4 Substrates beyond $300 \mathrm{~mm}$ (such as $450 \mathrm{~mm}$ ) in diameter will need to be introduced in order to manage the manufacturing costs of large chip size devices.

1.5 As supply voltage is scaled or reduced, cross-talk has become an issue for all clock and signal wiring levels; the near term solution adopted by the industry is the use of thinner copper metallization to lower line-to-line capacitance. Although copper-containing chips were introduced in 1998, copper must be combined with new insulator materials.

2. System-on-a-chip:

"Historically, the Roadmap has emphasized the technological limits of silicon production, leading to the specification of the most complex chips that can be developed in the categories of memory, microprocessor (MPU), and ASIC at a particular technology node. With the growing importance of high-volume consumer markets and the ability to integrate almost all aspects of a system design on a single chip, the Roadmap has included an additional vehicle to capture the requirements of this important, emerging area. We refer to this vehicle as a System-On-a-Chip (SoC). There are a number of characteristics that distinguish a mainstream SoC, but the main consideration is that it is primarily defined by its performance and cost rather than by technological limits. As a system-on-a-chip, these chips are often mixedtechnology designs, including such diverse combinations as embedded DRAM, high-performance or low-power logic, analog, rf, and even more esoteric technologies like MicroElectroMechanical Systems (MEMS) and optical input/output. In all categories of the Roadmap, design productivity is a key requirement. This is particularly true for the SoC category, where time-to-market for a particular applicationspecific capability is a key requirement of the designs."

information the size of $10 \mathrm{~nm}$ or smaller. In holographic storage, high-information density is achieved, for example, by storing in the same spatial spot but using different frequencies of light. Information is stored page by page. Although the concept of holographic storage is old, it has been limited by the availability of suitable storage materials, and this continues to bedevil researchers even now.

Scanning-probe storage, in which small clumps of atoms are used to record information, was demonstrated very shortly after the scanning tunneling microscope was developed. It has the limitation of speed in accessing the information stored on a medium. In order to circumvent this limitation, it was realized that an array of probes working in parallel could parallel the read-write process and thus obtain respectable read-write speeds. Such arrays, in which the tip-to-tip spacing is about $200 \mu \mathrm{m}$, have been built out of single-crystal silicon, using MEMS technology. The entire array of tips moves relative to the substrate, thus providing a means for writing or reading a block of information. Such arrays have been proposed for use not only for the "nanoCD," but also for nanolithography.

The scanning microprobes use a variety of different techniques to write and read information. The scanning tunneling probes use tunneling currents for heating or field evaporation for writing information and changes in tunneling currents for reading. Atomic-scale storage is possible, thus approaching densities of petabits / in. ${ }^{2}$. For example, vacancies in $\mathrm{MoS}_{2}$ were formed at selected sites, using field evaporation, and were used as bits of information. Alternatively, atoms can be deposited on substrates and these used as bits of information. The atomic force probes can be used on insulating substrates. Bits of information are written by modifying the surface. In one scheme, a gold film is deposited on a tip, and a field between the tip and substrate is used to peel off atoms and deposit them on the surface of the substrate. These can be read by the atomic force tip. The density of storage is less than the tunneling mode, for it is difficult to control the number of atoms that compose a cluster that form a bit. Even so, the density of storage is in the 100 gigabit/in. ${ }^{2}$ range. Similar densities can be obtained with near-field scanning optical microscopes using either MO or phase-change media. Somewhat lower but still-impressive storage densities have been achieved with either magnetic or electrostatic force probes. For example, 100-nm-sized domain reversals, corresponding to a storage density of 25 gigabits/in. ${ }^{2}$, were pro- 
duced by an electrostatic probe in a ferroelectric film. It is clear that in the future, we will use one or more of the scanning probe ideas to produce very high-density and very compact storage chips. There will be no spinning disks or flying heads. In human terms, a single disk with a petabit of storage provides approximately a movie a day for over 60 years.

\section{Memory/Microprocessor/ASIC/ Systems-on-a-Chip}

The worldwide semiconductor industry has established a roadmap for semiconductors. The latest version (1999) is now available at Web site www.itrs.net/ 1999_SIA_Roadmap/Home.htm. Unlike the three areas discussed thus far, the semiconductor roadmap provides a great deal of information on materials and processing needs for the next 15 years, assuming that the historical rate of progress must be maintained. The sidebar shows some highlights of the International Technology Roadmap for Semiconductors, selected for their relevance to this article.*

From the two points extracted and quoted from the Semiconductor Roadmap, materials clearly play a very important role. Equally clear, though, is the concern that we are approaching fundamental limits to scaling and, at the device level, to the historical increase in density, speed, and decrease in price. I believe that this limit is not likely to be visible to the user for a while because system performance will likely follow the historic trend through better design and system integration. However, from the perspective of materials research, the limits to device miniaturization offer challenges to propose alternative memory and processing devices. This is a unique period of opportunity for new ideas, for the mainstream technology will not continue to prevail against competitive ideas, as it has in the past.

A reasonable approach for the near term might be to combine silicon-based semiconductor technology with new ideas and produce hybrid devices-for example, a silicon-based processor with memory placed directly above the plane of the microprocessor. The memory plane is made by a technology that is compatible with the silicon processing-for example, molecular or organic memories. Such memories, as shown in the laboratory, can have very high densities. Single electron (Coulomb blockade), spin electronics, polarized electron tunneling (magnetic

\footnotetext{
*Semiconductor Industry Association. International Technology Roadmap for Semiconductors: 1999 edition. Austin, TX:International SEMATECH, 1999.
}

random-access memories), and phase change induced by current pulses are some of the ideas that are being explored in current research. There are also proposals to use HTSs in conjunction with silicon technology to produce hybrid devices or, alternatively, all superconducting devices in which Josephson coupling is controlled by a gate or, for logic applications, rapid single-flux quantum devices. Based on the Semiconductor Roadmap, materials clearly plays an important role.

From the perspective of
materials research, the limits
to device miniaturization
offer challenges to propose
alternative memory and
processing devices.

\section{Future: A Gleam in the Eye}

A characteristic feature of all of the device ideas discussed here is the binary bit of information, as the fundamental unit of transaction, and a predetermined layout that is designed by engineers and implemented by process technology, including lithography. This has been the historical path and a proven path. It is not clear that this will continue to be so into the long-term future. There are several "out-of-the-box thinking" possibilities.

Quantum computers manipulate coherent quantum states, made up of an arbitrarily large superposition of, say, two states such as spin up or down. The latter is the quantum equivalent of the classical binary bit as, for example, in the case of magnetization pointing up or down in magnetic storage. For this reason, it is called a quantum bit or a qubit. The superposition enables the quantum computer to calculate, for certain algorithms, what a conventional and massively parallel machine may do, but in times that are orders of magnitude less. There is one serious drawback. A quantum computer is very susceptible to decoherence by interaction with the external world. Remarkably enough, it has been shown that, within limits of decoherence, error correction codes exist, similar to their counterparts in classical systems. The objective of research in recent years has been to find a solid-state material in which quantum computing can be demonstrated beyond a few qubits.

A number of decades back, well before the advent of silicon technology, it was demonstrated by Turing that a universal computer could be built if one had a device, such as a tape, for recording bits of information and a machine that could act on these bits to change them, following simple instructions. Less than 10 years ago, it was realized that a DNA molecule, which stores the blueprint of life, could be used to store information, akin to the tape, and that enzymes such as polymerases and ligases operate on this information. Using this notion, it was demonstrated that a particular problem in computer science, called the Hamilton path problem, could be solved. Using a very large number of replicated DNA molecules, the presence of the desired sequence was obtained by eliminating all others that did not have the right sequence. This demonstration has initiated research into using DNA to demonstrate its viability as an alternate to silicon technology for processing information, not just in DNA floating in solution in a test tube but also in DNA tethered onto predetermined sites on a chip.

The observation that a single molecule, such as DNA, can store a four-bit code that can be replicated, and that segments of this replica subsequently can generate and control a great variety of forms and functions is very well known by beings that were produced in this fashion. Can the same beings synthesize a molecule that, like the DNA molecule, produces objects that perform useful functions, including elements of information technology? I believe the answer is yes. It seems to me that this will evolve from research currently under way in two areas. These areas are life sciences, combined with information sciences, and "quantum legos," a term I define next.

There is a rapidly growing realization that the interface between information technology and the life sciences is a very fertile field. Let me touch on a few ideas. The interaction of neurons or their electrical signals with appropriately designed sensors or transmitters and, at a macroscopic level, sensors that sense or transmit the emotional state of a human as manifested by changes in the physical body is an example that is currently being explored. Information technology will be widely used to communicate and control the physical state, including abnormalities associated with disease, by biochips, which will include not just biologically active sensors, but also circuitry for processing and transmitting by wireless means. This transmission, like radioactive isotopes, will localize the physical location of the biologically active site and, either by preprogrammed means or by an external signal, cause the chip to deliver drugs. 
There is some work in progress in this area, but it is still in a very early phase. All of the knowledge and expertise generated in technologies associated with communications, very high density integrated circuits, and novel power supplies, including those that rely on the functioning of a human body, will be used and combined with materials issues concerned with biocompatability. As this work progresses and familiarity grows between the biological and information sciences, a new discipline will evolve. At the root of this evolution will be "quantum legos."

Atomism, or more generally, reductionism, as a philosophy enunciated by the ancient Greek and Indian philosophers, has served science well. The drive to reduce an apparently complex observation to its conceptual elements is now deeply rooted in our training as scientists. One of the consequences of this philosophy has been to encourage partition of our knowledge into bureaus such as biology, chemistry, physics, and materials. This has served us remarkably well, for we can train scientists to go deep into their disciplines. Underlying all of these diverse disciplines is a handful of different atoms. They combine in myriad ways to produce most of the physical world we deal with. Engineers, in contrast to most scientists, are synthesizers or integrators. They take diverse elements and put them together to create a composite that provides a desired functionality: a computer, a car, or an airplane. We are now reaching a state of development in our understanding and in our ability to manipulate individual atoms
We shall use quantum entities, such as atoms and clusters of atoms, to build molecules that replicate, synthesize, and carry out other functions, much as

DNA does for life.

or molecules that this distinction will, I believe, be moot. We shall use quantum entities, such as atoms and clusters of atoms, to build molecules that replicate, synthesize, and carry out other functions, much as DNA does for life. A good part of the future information technology or, for that matter, technology of interest to other industries, will emerge from these quantum building blocks or "legos."

The information technology of the future, say, toward the end of this century, will bear little resemblance to the gadgets we use today. Only the underlying human needs, such as to communicate with each other, to learn, to be entertained, or to be informed, will be the constants; that and the laws of the universe.

Praveen Chaudhari received his doctoral degree from the Massachusetts Institute of Technology in 1966. He joined IBM the same year and over the years carried out his personal research. He held various management responsibilities in science and in technologies such as optical storage, semiconductors, magnetic bubbles, Josephson tunneling, and liquidcrystal displays. Chaudhari was appointed Director in 1981 and Vice-President, Science, in 1982. In this capacity he was responsible for science programs at Almaden, Yorktown, and Zurich. During this period, the science programs at the IBM Research Laboratories grew significantly and IBM scientists were awarded Nobel Prizes for two consecutive years (1986 and 1987). In 1989, Chaudhari became a member of the Corporate Technical Committee, and in 1990, he returned to the Research Division as a Research Staff Member.

In the United States, Chaudhari was executive secretary of President Reagan's Advisory Council on Superconductivity (1988), and served as a member of the National Commission on Superconductivity that reported its findings to President Bush (1989). He has also served on the U.S. National Critical Technologies Panel (1992 and 1993). Chaudhari received the National Medal of Technology awarded by President Clinton (1995). In 1988, in India, he reported to Prime Minister Rajiv Gandhi on science and technology, and in 1993, at the request of the Indian Minister for Sciences and Technology, led an IBM group to evaluate the Indian parallel-computer activities, and in 1994 made a presentation to Prime Minister Rao of India on Materials and Critical Technologies.

Chaudhari has published over 150 technical papers and holds over 20 patents. He has served on numerous university and publicpolicy committees and advisory boards, and has received many honors for his research and personal contributions to science and the management of science. He is a member of the National Academy of Engineering and the American Academy of Arts and Sciences, a Fellow of the American Physical Society, and a member of the Third World Academy of Sciences.

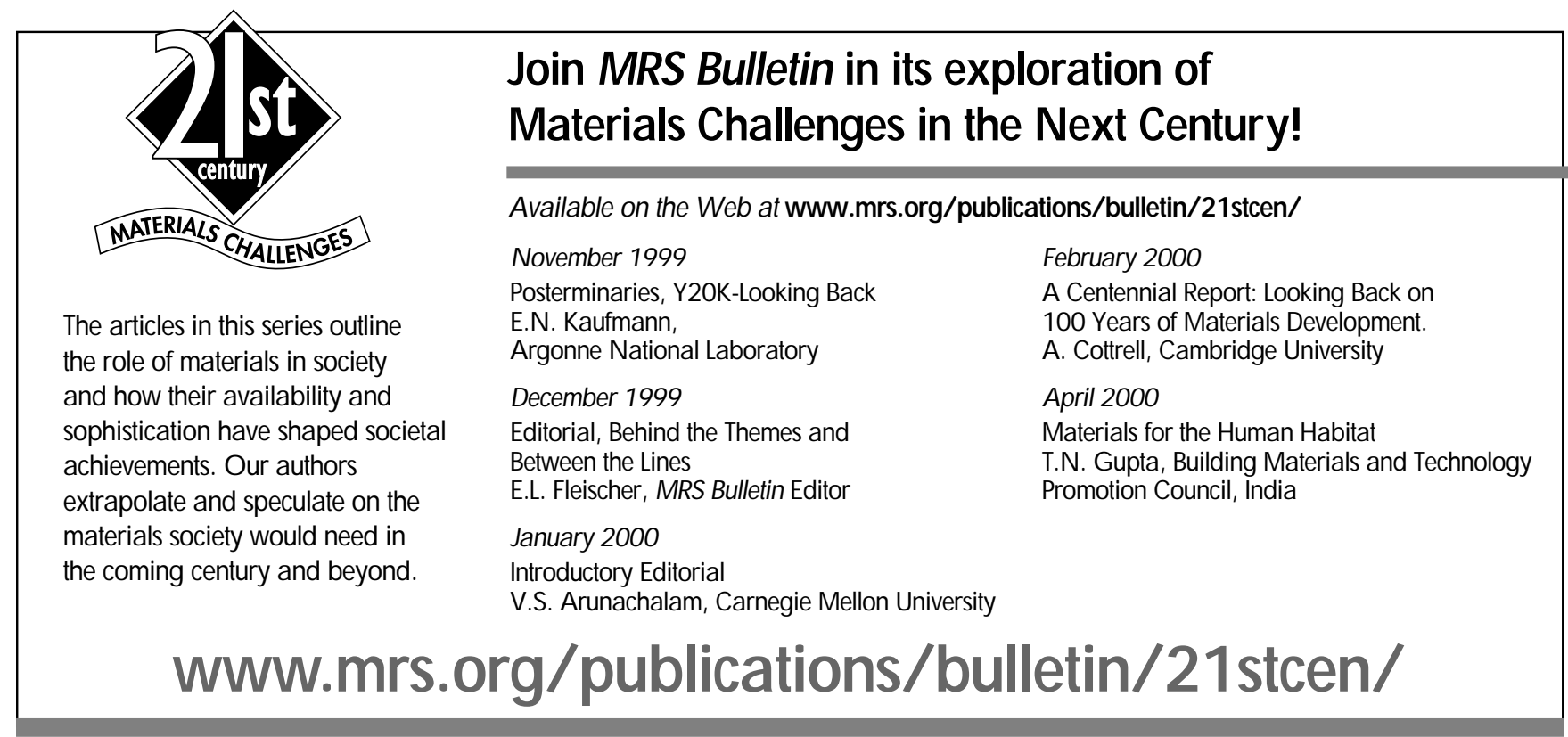

